\title{
Normocomplementemic Idiopathic Urticarial Vasculitis
}

\author{
Matheo Augusto Morandi Stumpf
}

\begin{abstract}
(c) (1) (2) (2)
Copyright: (C) 202

DOI: https://doi.org//0.3329/jom.v22il.5/395

Copyright: (c) 2021 ......... This is an open access article published under the Creative Commons AttributionNonCommercial-NoDerivatives 4.0 International License, which permits use, distribution and reproduction in any medium, provided the original work is properly cited, is not changed in any way and it is not used for commercial purposes.
\end{abstract}

Received: 10 October, 2020;

Accepted: 13 November, 2020

A 17-year-old-woman presented to the internal medicine outpatient clinic with a single pruritic erythematous plaque in her left cubital fossa (Figure-1A). The lesion had a 2 days evolution. The systemic physical exam had no other abnormalities. She denied drug use, comorbidities and other systemic symptoms. The only different thing she stated was the use of new brand soap about a week ago. A diagnosis of hive/simple urticaria was made empirically and she was given an antihistamine and a topical corticosteroid. However, one week later she came back with disseminated pruritic erythematous lesions.

On this new examination, the larger lesions had some purpuric component in the center (Figure-1B) with positive diascopy. Erythema multiforme-like lesions were present in her hands as well (Figure-1C). Since no improvement was observed, a differential of urticarial vasculitis was proposed. A biopsy was made to confirm the diagnosis.

Urticarial vasculitis is a leukocytoclastic vasculitis with wheals that usually stay more than 24 hours and leaves small patches of brownish color due to micro bleedings ${ }^{1}$. Vasculitis components may be present such as palpable petechiae, diascopy positive for blood extravasated and purpura. Three clinical entities may be present: normocomplementemic urticarial vasculitis (NUV), hypocomplementemic vasculitis and hypocomplementemic urticarial vasculitis syndrome. NUV is typically self-limited, idiopathic and benign. The other two generally have more systemic symptoms and are recurrent ${ }^{2}$.

Other differentials that need to be considered are chronic spontaneous urticaria, lupus, cryoglobulinemia, AHA syndrome (arthritis, hives, urticaria), Schnitzler syndrome

Department of Medicine, Ponta Grossa State University, Ponta Grossa, Brazil.

Corresponding author: Matheo Augusto Morandi Stumpf, Adress: Av. Carlos Cavalcanti, 4748, Medicine Department, Uvaranas CEP: 84030-900, Ponta Grossa, Brazil. Phone: (42) 32203793.

E-mail: matheoaugusto@hotmail.com and genetic disease such as Muckle-Wells syndrome ${ }^{2}$. A biopsy is generally warranted. However, a recent article from García-García stated that the classic clinical features (persistence of wheals over 24 hours and presence of purpura or residual hyperpigmentation) have a specificity of $97.2 \%$ for the diagnosis of urticarial vasculitis ${ }^{3}$ and perhaps a biopsy in those cases would be unnecessary.
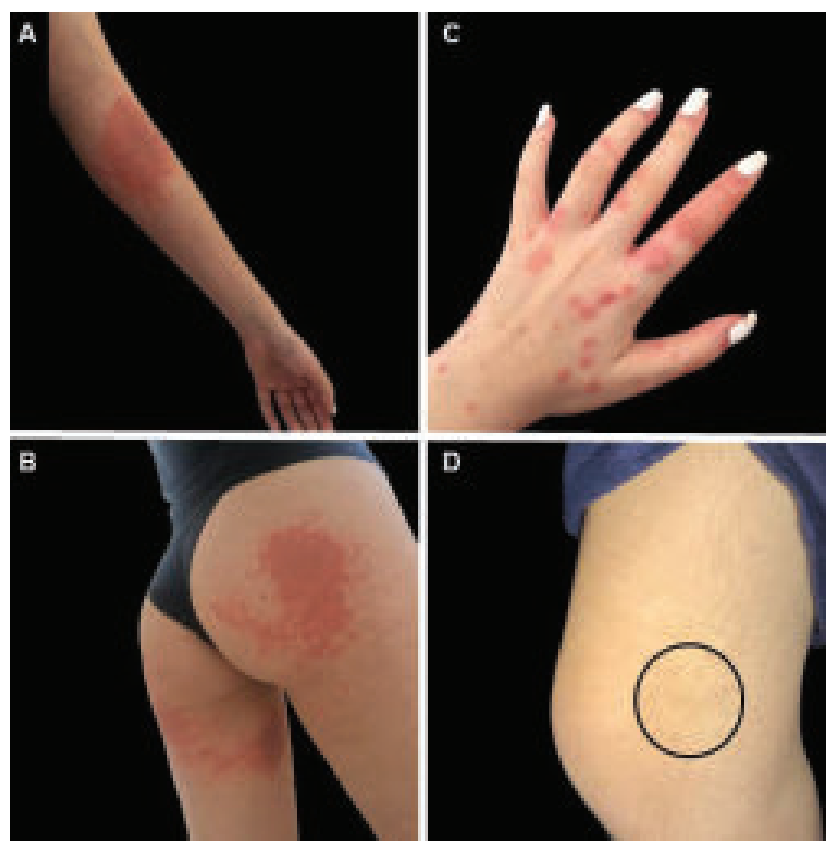

Figure 1: $A$-Simple hive in left upper limb; B - More disseminated lesions with purpura; $C$-Erythema multiformlike lesions in hand; $D$-Residual hyperpigmentation more evident inside the marked circle

Treatment for NUV is generally with corticosteroids and antihistamine for pruritus control, with great recovery. In the case of hypocomplementemic urticarial vasculitis due to recurrence, an immunosuppressive drug is usually prescribed, such as dapsone, hydroxychloroquine or 
cyclophosphamide. Investigation for secondary causes (such as autoimmune diseases, malignancies, infections) and its treatment is warranted as well ${ }^{4}$.

Our patient was treated with prednisone $1 \mathrm{mg}$ per day and antihistamines were maintained for pruritus control. After a week with corticosteroids, she had a full recovery, maintaining minimal residual hyperpigmentation typically seen in resolved urticarial vasculitis (Figure-1D). We opted to not start a chronic immunosuppressive treatment because it was her first episode. Moreover, her blood test screening for secondary causes of urticarial vasculitis was all negative (ANA, ANCA, complement, rheumatoid factor, hepatitis B, $\mathrm{C}, \mathrm{HIV}$ and syphilis). After a year of follow up she did not demonstrate any new episodes of urticarial vasculitis.

\section{References}

1. Zuberbier T, Maurer M. Urticarial vasculitis and Schnitzler syndrome. Immunol Allergy Clin North Am. 2014; 34(1):141-7.

2. Hamad A, Jithpratuck W, Krishnaswamy G. Urticarial vasculitis and associated disorders. Ann Allergy Asthma Immunol. 2017; 118(4):394-398.

3. García-García B, Aubán-Pariente J, Munguía-Calzada P, Vivanco B, Argenziano G, Vázquez-López F. Development of a clinical-dermoscopic model for the diagnosis of urticarial vasculitis. Sci Rep. 2020; 10(1):6092.

4. Kolkhir P, Grakhova M, Bonnekoh H, Krause K, Maurer M. Treatment of urticarial vasculitis: A systematic review. J Allergy Clin Immunol. 2019; 143(2):458-466. 